\title{
Freezing in Sealed Capillary as an Alternative to HPF
}

\author{
S. Yakovlev and K. H. Downing
}

Lawrence Berkeley National Laboratory, Berkeley, CA 94720

Cryosectioning of frozen hydrated biological materials is a well established technique that allows preparing TEM samples of biological objects in a near native state. In contrast to cryoplunging, it allows examining slices of objects that are too thick to be electron transparent. Successful cryosectioning largely relies on the quality of cryofixation of the bulk biological material. Since fixation at ambient pressure results in high quality cryofixation of only a thin surface layer, high pressure methods are typically used [1].

Freezing in a constant volume [2-4] is an attractive alternative to conventional high pressure freezing (HPF). Among advantages of this method are simplicity of use and no need for dedicated equipment. Samples may be frozen in standard for HPF copper capillary tubes [3]. Moreover the method allows use of less cryoprotectant for amorphization of high water content samples than conventional HPF does [4]. For biological samples containing gas bubbles (e.g. most plants) the method provides an additional advantage by allowing a slight pressure increase prior to freezing to avoid artifacts caused by bubble implosion.

In this work we present evidence that amorphization of the sample in a sealed capillary tube is promoted by both added cryoprotectant agents and pressure buildup due to formation of low density crystalline ice in part of the sample. We examined aqueous dextran solutions with a range of concentration. In standard capillary tubes only samples that contained over 10 percent cryoprotectant produce any amorphous ice. In samples containing less than 30 percent dextran only the central part becomes amorphous. Figure 1 presents an optical image of a frozen and trimmed sample containing 15 percent dextran, and x-ray diffraction patterns collected from the central amorphous and peripheral crystalline regions of the sample. The amorphous ice is in the high density phase, as independently supported by the diffraction pattern and visual observation of the higher refractive index in the central part of the sample. The crystalline ice has a cubic crystal lattice and extremely small size of the crystallites.

It is clear that both freezing in a sealed tube and conventional HPF methods are capable of achieving similar pressures (limited by the strength of the tube) and similar rates of heat extraction. However freezing in a sealed capillary has a lower dextran content requirement, which may be explained by a higher rate of heat transfer through the crystalline ice due to the higher thermal diffusivities of both hexagonal and cubic ices than water or amorphous ice. This difference may be demonstrated by modeling of the heat transfer. Figures 2 shows numerical solutions of the heat transfer equations for a cylindrical sample of water rapidly cooled from $20{ }^{\circ} \mathrm{C}$ to $-170{ }^{\circ} \mathrm{C}$. One solution corresponds to the case where water crystallizes and explicitly includes latent heat released upon crystallization. The other solution, corresponding to complete vitrification, is obtained using extrapolated thermodynamic parameters of water. It is clear that the crystallizing sample cools much faster than the vitrifying one.

Solution of the heat transfer equation shows that the cooling rate as the temperature passes $0{ }^{\circ} \mathrm{C}$ in the center of the crystallizing sample may be more than two orders of magnitude higher than in the case of perfect vitrification. This result may have important consequences. Isolated cells that have lower water content than the medium and therefore crystallize at a lower temperature may cool at very high rates and vitrify even when the surrounding medium crystallizes. Figure 3 shows a section 
of a bacterium frozen in 10 percent dextran medium. The medium clearly has a segregation pattern characteristic of water crystallization. At the same time the internal part of the bacterium appears relatively well preserved.

We conclude that freezing in a sealed capillary tube is not only a simpler and less expensive alternative to conventional HPF but also has the potential for achieving better cryopreservation due to higher cooling rates.

\section{References}

[1] Dahl, R. and L.A. Staehelin, High-pressure freezing for the preservation of biological structure: Theory and practice. J Elect Microsc Tech 13(3): p. 165-174, 1989.

[2] Szobota, S.A. and B. Rubinsky, Analysis of isochoric subcooling. Cryobiology 53(1): p. 139-142, 2006.

[3] Leunissen, J.L.M. and H. Yi, Self-pressurized rapid freezing (SPRF): A novel cryofixation method for specimen preparation in electron microscopy. J Microsc 235(1): p. 25-35, 2009.

[4] Yakovlev, S., B. Luef, L. R. Comolli, K. H. Downing, Self Pressure Freezing of Caulobacter crescentus for the preparation of frozen hydrated sections. Microsc Microanal 16(Suppl. 2): p. 860-861, 2010.

[5] The authors are grateful to Dr. Luis Comolli for useful suggestions on this project. This work was supported by Director, Office of Science, Office of Basic Energy Sciences, Materials Sciences and Engineering Division, U.S. Department of Energy, under Contract Number DE-AC02-05CH11231
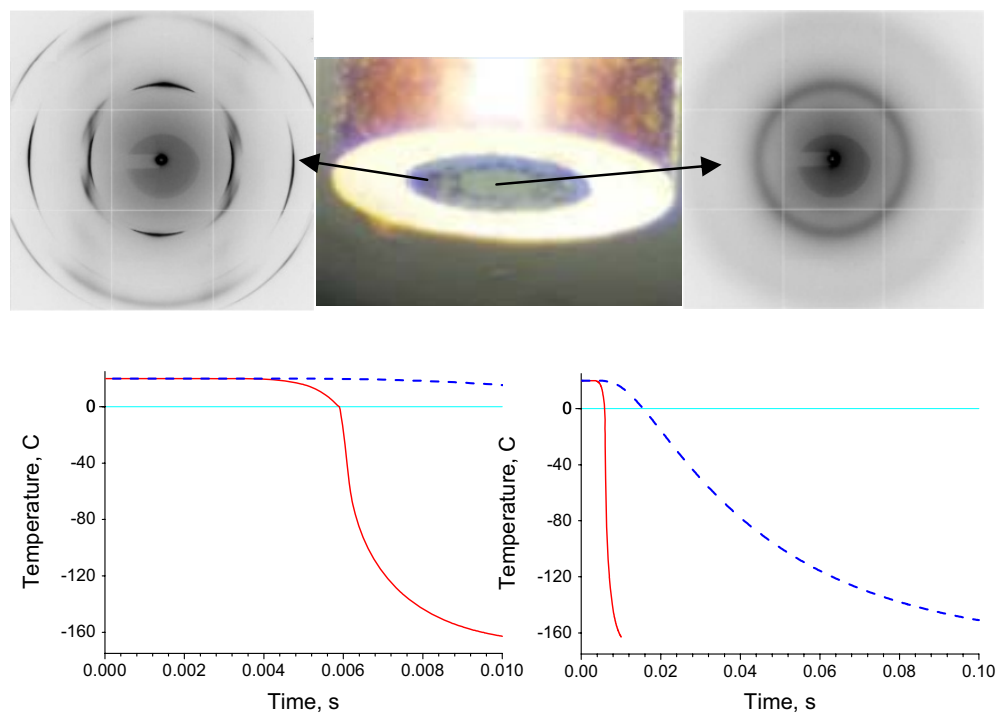

FIG. 1. Center: optical image of $15 \%$ dextran sample frozen in a copper capillary. Central glassy part is HDA ice and rough material on periphery is cubic ice. images at left and right are synchrotron $\mathrm{x}$-ray diffraction patterns from marked regions.

FIG. 2. Numerical solution of the heat transfer equation showing temperature in the center of a capillary for complete crystallization (solid red line) and complete vitrification (dashed blue line). Note different scales on time axes.

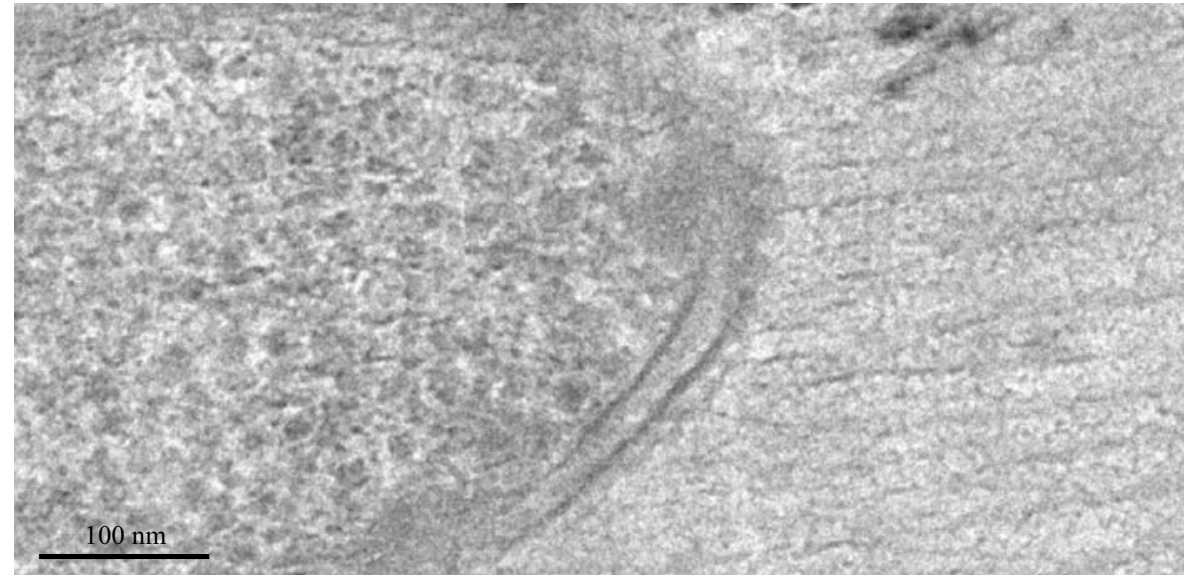

FIG. 3. Section of Caulobacter crescentus bacterium frozen in $10 \%$ dextran medium. Medium clearly show segregation pattern while internal part of bacterium is well preserved. 